\title{
A Framework for Function Allocations in Intelligent Driver Interface Design for Comfort and Safety
}

\author{
Wuhong Wang *, Fuguo Hou, Huachun Tan \\ Department of Transportation Engineering, Beijing Institute of Technology \\ Beijing 100081, P. R. China \\ Heiner Bubb \\ Institute of Ergonomics, Technical University of Munich \\ Munich, D-85747, Germany
}

Received: 25-10-2009

Accepted: 02-06-2010

\begin{abstract}
This paper presents a framework for ecological function allocations and optimization matching solutions for a human-machine interface with intelligent characteristics by "who does what, when and how" consideration. As a highlighted example in nature-social system, intelligent transportation system has been playing increasingly role in keeping traffic safety, our research is concerned with identifying human factors problem of In-vehicle Support Systems (ISSs) and revealing the consequence of the effects of ISSs on driver cognitive interface. The primary objective is to explore some new ergonomics principals that will be able to use to design an intelligent driver interface for comfort and safety, which will address the impact of driver interfaces layouts, traffic information types, and driving behavioral factors on the intelligent vehicles safety design.
\end{abstract}

Keywords: Function allocation, Human-machine system, Driver cognitive interface, In-vehicle support system.

\section{Introduction}

Since modern information and communication devices are changing the characteristics of high-level information processing of human operator, the growing complexity of the nature-social system such as computer networks, intelligent transportation system (ITS), makes operators to understand the systems internal function increasingly difficulty. Therefore, it is necessary to the human operator controls the hazardous processes work with interpretation of the situation and decision making ${ }^{1-3}$.Because all possible events that might happen during the expected lifetime of the system operation usually could not be predicted in the design phase, it is very important to determine what human operator to does and what machine to does.

According to basically various ways of representing the constraints in the characteristics of a deterministic system, human operator performance can be distinguished by three typical behaviour, they are respectively skill-based, rule-based and knowledgebased behaviour ${ }^{4-6}$.A skill-based behaviour represents a type of behaviour that requires very little or no conscious control to execute an action once an intention is formed. The performance is smooth, automated, and consists of highly integrated patterns of behaviour. A rule-based behaviour is characterized by the use of rules and procedures to select a course of action in a familiar

*Corresponding author: wangwuhong@bit.edu.cn 
work situation. The rules can be a set of instructions acquired by the operator through experience or given by supervisors. A knowledge-based behaviour must be employed when the situation is novel and unexpected. The operators are required to know the fundamental principles by which the system is governed. Since operators need to form explicit goals based on their current analysis of the system, cognitive workload in knowledge-based behaviour is typically greater than the one in skill-based or rule-based behaviours ${ }^{7-9}$.

At a higher level of conscious planning for machine running safety, human operators are not simply deterministic input-output devices but also goal-oriented creatures that actively select their goals and seek the relevant information, so we need systematic descriptions of human performance in total from the observation of information to the physical actions ${ }^{10-11}$. As a matter of fact, traditional function allocation and adaptive function allocation could not meet all requirements of function allocation and optimization matching solution between human operator and machine while the new working environment is really constructed $^{5,9,11}$.

Although considerable efforts have been required to apply these function allocation strategies to solve driving safety problems, Intelligent Transportation Systems (ITS) clearly represent an increase in the number of displays and controls for the driving environment in vehicle cab, along with a concurrent increase in the amount and complexity of information presented to the driver ${ }^{12-14}$. If human factors integration and design issues are not addressed throughout the development process for In-vehicle Support Systems (ISSs), there is a risk that will lead to information overload, driver confusion, and actual decreases in driving safety ${ }^{15}$. As a result, the role of human factors is widely acknowledged to be critical to the successful implementation of ITS ${ }^{16-18}$. However, too little research is directed toward advanced human-ITS interaction. This is due to these design practices are mostly based on SSSI (Single Sensor, Single-Indicator) principals. Consequently, a function allocation strategy will be created with a novel consideration for addressing the issues of human factors integration and design in ITS.

This paper focuses on analysis of human factors in function allocation because the usability of humanmachine interface will influence safety of the machine operation. The emphasis of our work is concerned with investigating the effects of In-vehicle Support Systems (ISSs) upon optimization matching in intelligent drivervehicle interface design. Especially a conceptual framework for ecological function allocation is proposed by "who does what and when and how" consideration.

\section{Human Machine Interface and Function Allocations}

Human-machine interface is the place where the operator interacts physically and mentally with the machine, it is about behavioral characteristics through the cooperation way of operator and machine. Since human-machine interface is characterized by all events, which are familiar, unfamiliar but anticipated, unfamiliar and unanticipated to operators, it is important that a machine can provide operator with the information needed to cope with such events.

During human-machine interface design process, function allocation is the design decision to determine which functions are to be performed by human and which functions are to be performed by machine for achieving the required system goals (Fig.1). Various strategies for function allocation in human-machine interfaces have already been proposed by applying the principals of human factor and ergonomics ${ }^{5,9,30}$.

The first is the traditional function allocation. In general, traditional function allocation strategies can be classified into three types: comparison allocation, leftover allocation and economic allocation. Comparison allocation is about what human is better and what machine is better, the strategies of this type compare relative capabilities of human versus machine for each function, and they allocate the function to most capable agent (human or machine). Leftover allocation has focused on allocating machine every function that can be automated, and thus human operators are assigned the leftover functions to which no automation technology is available. Economic allocation tries to find an allocation ensuring economical efficiency. In fact, if automating the function is not cost-effective, the function is assigned to the operators. The traditional function allocation depends on the "who does what" design strategy, such decision yield function allocations that are static, it mean that once a function is allocated 
to an agent, the agent is responsible for the function at all times.

The second is the adaptive function allocation that consider "who does what and when". It is dynamic in function allocation. Since system condition may change with time, human operator behaviour will degrade gradually because of psychological or physiological factors, it is necessary to reallocation functions between the human operator and machine dynamically so as to keep machine running safety.

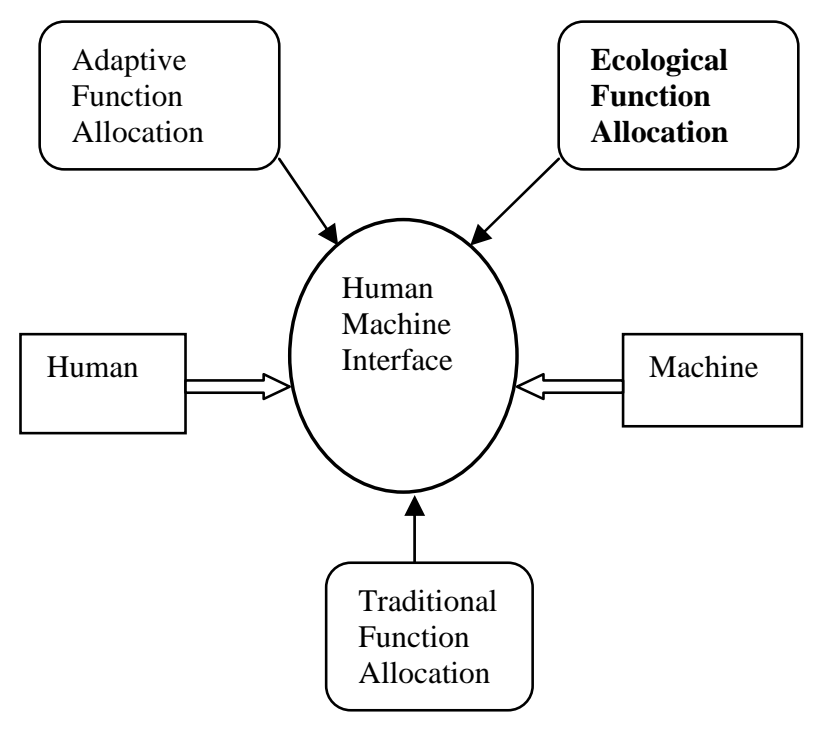

Fig. 1. Function allocations in human-machine interface design process

\section{Driver Behaviour Analysis in Intelligent Transportation Systems}

A highlighted example in the nature-social system is Intelligent Transportation Systems (ITS), which applies state-of-the-art information technologies(i. e., digital maps, communication links, microprocessors, sensing and image processing) to provide more efficient and effective solutions to current traffic congest and traffic safety problems ${ }^{19-21}$. While effectively integrated and deployed, ITS technologies could offer many benefits to the public. For example, it could help drivers to control vehicles more safety, and it might improve the efficiency, productivity and safety of existing traffic facilities to increase the mobility of travelers, especially to alleviate the impact of traffic on the environment. In addition, ITS and its sub-systems could provide various levels of traffic information and trip advisory to traffic users so that travelers can make timely and informed travel decisions ${ }^{22-23}$.

High-level information processing is dependent on individual driving characteristics very much in intelligent traffic information network environment. Because of driver physical and psychological limitations, driver always does not perceive information considerately, sometimes drivers commit some errors and the ISSs have unreliable factors, those might precipitate traffic accidents. The function of driver is to act on the steering and speed controls of the vehicle in order to travel under given road environment, meanwhile ISSs will maintain operating safety states for the advanced vehicle. The interactions of the intelligent road traffic information network environment and the driver are nearly always filtered through the ISSs. In this sense, the ISSs and driver's linkage with the intelligent road traffic information network environment will mostly consist of his positioning the vehicle, and the ISSs and driver's influence over the advanced vehicle through a limited number of cooperation controls.

Driver-vehicle interface must be designed carefully to let driver knows what he will do, why he does that, or what he is going to do next. ISSs as one major area of ITS development, it will provide the primary means by 
which drivers will interact with ITS. Thus, determination of the man-machine system characteristics of ISSs is critical to the success of the ITS, and the ISSs provide some new interfaces among the driver, the vehicle and road traffic environment and have immense potential to enhance traffic safety. Regardless of the nature of the ISSs under development, there are certain key human factor issues that are common to the design of most types of man-machine interface of ISSs. For example, when designing and implementing the locus of adaptive control, some items such as ecology interface layout, real-time information content and presentation, type of information display, timeliness of information will need to be paid much more attentions. Accordingly, we should take key human factors in account not only information potentially available to drivers but also the roles of the ISSs.

\section{Impacts of ISSs on Optimization Matching of Driver-Vehicle Interface}

According to role of "intelligence" to complement the driver, ISSs can be considered as falling into the three types:

(i)sub-systems that directly impinge on the driving task (i.e., collision/obstacle avoidance devices, lane-keeping devices, gap warning devices, intelligent cruise control devices, intelligent maneuvers devices, vision enhancement devices),

(ii)sub-systems that provide information related to components of the road environment, vehicle or driver (i.e., traffic monitoring and control systems, vehicle condition monitoring systems, route guidance systems), (iii)sub-systems that are unrelated primary driving task(i.e., cellular phone, portable computer, fax machine).

ISSs may place message screens, collision avoidance displays and much more in the new generation vehicle. The application of ISSs will not only lead to the increase of display number in vehicle cab but also expand the driving requirements to driver's ability. Since the information overload will lead to increase in the number of displays and controls, increase in the amount and complexity of information presented to the driver, it is easy to make driver confusion because of traffic information overload ${ }^{13,15,20,28}$. The reason for this is that the inherent complexity of in-vehicle auditory and visual information, the widely ranging knowledge, skills, and abilities of the driving population.

\subsection{Increase in the number of displays and control for the driving environment}

Because of considerable amount of information "communication" between driver and vehicle, it not only increases information burden of driver-vehicle interface, but also makes operation areas of cab become more and more crowed. For example, if the number and variety of ISSs is continuously increasing in the cab of vehicle, the vehicle will need to add about 25 types of new monitors and controls functions, and also require adding about $400 \mathrm{~cm}^{2}$ areas for instrument panel.

Although current vehicle has contained kinds of apparatus with operation function, driver must face no less 10 kinds of new devices and ISSs which driver is not familiar with their function and will be strictly required to operate. On the one hand, these in-vehicle support systems have the potential to enhance comfort, efficiency and even safety of the driving task. On the other hand, ergonomists and psychologists are warning against distraction effects and information overload caused by performing additional tasks while driving.

\subsection{Concurrent expansion of the driving skill requirements to driver}

The application of ISSs needs driver care for more and more information, which easily distracts driver's attention. As far as these common drivers are concerned, at the same time when they drive vehicles en-route, they are not easy to use, monitor, inspect and understand the information. Because urban traffic flow is with fixed characteristics, drivers need momentarily deal with variously sudden traffic incidents on the road. So, even though driver can complete the above operations, driver's attention also may so excessively distract.

Driver receives information about driving environment not as a concise list of symbols, but as a constantly changing set of sensor readings. Since time constraints prevent processing all of this information at every time instant, driver must intelligently select the information most critical to the immediate driving task. With driving 
complexity increases under highly congested urban traffic conditions, any additional task becomes very disruptive of driving behaviour. For example, the use of cellular telephone has been found to be a potent

\subsection{Inherent complexity of in-vehicle auditory and visual information}

Correctly choosing the appropriate sensory modality (auditory, visual, or tactile) to use for the delivery of invehicle information is an important task to consider while designing a driver-vehicle interface. Map information from in-vehicle navigation display also could be not neglected when the driver is actually driving through an intersection. As a result, sensory modality allocation can greatly affect both the vehicle safety and usability of driver-vehicle interface. For example, excessive amounts of visual information can overload the visual modality that already provides about $80 \%$ of driving information. The use of in-vehicle auditory information can lead to driving environment that is unusable, frustrating, annoying, and even dangerous.

In general, when designing a driver-vehicle interface, sufficient time must be allowed for the driver to respond to any delivered auditory or visual information. On the one hand, if the new functions and traditional functions cannot be harmonized reasonably on the instrument panel of vehicle, the panel will be very crowded; On the other hand, if this problem can be resolved by new principles of the driver-vehicle ergonomics design, the vehicle must suit to the driver and the driver can adapt to the vehicle.

Driver needs time to receive the information, decide whether it is relevant, and act on it. It is recommended that 95th percentile to 99th percentile driver population should have ample time to respond to in-vehicle information under most driving circumstances, namely, we should follow these steps:

(i) All control components and monitors must be placed within the range that drivers can reach easily;

(ii) The functions which are often used should be set on the place that is nearer to the driver;

(iii) There should be enough space between kinds of operation appliances so as to distinguish and operate; disrupter of driving behaviour. Under this circumstance, expansion of the driving skill requirements would lead driver could not devote his full attention to process the traffic information at the appropriate time.

(iv) The design layout of the instrument panel should benefit to driving safety in any way.

When we design a driver-vehicle interface, if we could use these current delightful design methods and new principals, it can partly reduce the heavy information burden of driver-vehicle interface, but also it could radically realize the integration and synthesis of human factors in digital driving operation.

\section{Conceptual Framework for Ecological Function Allocation}

\subsection{Driving tasks}

As drivers are the most complicated core part of the traffic system, they have to perform the task of information processing, decision-making, vehicle adjusting and control almost at the same time. They firstly obtain traffic information from road environment and vehicle running conditions, then feed all the correlative rules to their brain and make decisions instantly. However, it is the most requiring job for drives to be ready at any minute for the latent danger that might suddenly show up in the driver's field of view and will almost at once attract all attention. As a result, some advanced assistant systems have been designed to support drivers in maintaining some safety thresholds or ensuring compliance with some formal driving rules (e.g. maintaining safe time headways in car-following situations).

In traffic operation driving task is a hierarchical process with three levels: control/operational, guidance/tactical and navigation/strategic level, it will be underlying cognitive control of driving ${ }^{14,17,19}$.The control/operational level consists of the immediate vehicle control inputs, which are largely automatic actions such as steering, braking, and shifting. The guidance/tactical level involve negotiation of common driving situations such as curves and intersections, gap acceptance in overtaking or entering the traffic flow, and obstacle avoidance. The navigation/strategic level 
involve general trip planning that includes setting trip goals, selecting routes and evaluating the costs.

The control hierarchy of driving tasks ${ }^{14,17}$ has been shown in Table 1. The horizontal row corresponds to the hierarchy of driving tasks, each category being roughly related to a time constant for driving task duration, it is described by three levels: navigation/strategic level means minutes to hours, guidance/tactical level means seconds, and control/operational level means milliseconds.

The vertical column corresponds to the levels of performance of drivers, it includes three type behavior: Skill-based driving behavior refers to driving behavior, which process familiar traffic events; Rule-based driving behaviour refers to driving behaviour, which process unfamiliar but anticipated traffic events; Knowledge-based driving behaviour refers to driving behaviour, which process unfamiliar and unanticipated events. For example, for the experienced drivers, most driving tasks cluster in the three cells from the upper left to the lower right and in the lower left; for the novice driver, they are mainly in the upper right. In general, skill-based driving behaviour is involved at the control/operational level, rule-based driving behaviour at the guidance/tactical level, and knowledge-based driving behaviour at the navigation/strategic level.

Table 1 Classification of selected driving tasks.

\begin{tabular}{|l|l|l|l|}
\hline & $\begin{array}{l}\text { Navigation/Strategic level of } \\
\text { driving task(Minutes) }\end{array}$ & $\begin{array}{l}\text { Guidance/Tactical level of } \\
\text { driving task(Seconds) }\end{array}$ & $\begin{array}{l}\text { Control/Operational level of } \\
\text { driving task(Milliseconds) }\end{array}$ \\
\hline $\begin{array}{l}\text { Knowledge-based driving } \\
\text { behaviour }\end{array}$ & Navigating in unfamiliar area & Controlling a skid on icy roads & Novice on first lesson \\
\hline $\begin{array}{l}\text { Rule-based driving } \\
\text { behaviour }\end{array}$ & Choice between familiar routes & Passing other vehicles & Driving unfamiliar vehicle \\
\hline $\begin{array}{l}\text { Skill-based driving } \\
\text { behaviour }\end{array}$ & Route used for daily commute & $\begin{array}{l}\text { Passing familiar junctions/ } \\
\text { intersection/ }\end{array}$ & Vehicle handling on curves \\
\hline
\end{tabular}

\subsection{Driving situation awareness}

The competence to a task-specific understanding of the working situation is termed as situation awareness ${ }^{2,8}$. Situation awareness was first presented in connection with pilot performance in air-to-air combat and the ability of commercial airline pilots to fly in difficult air traffic condition ${ }^{24}$. In general, driving like flying can be thought of as a dynamic control system, in which system input variables change over task time. The input variables such as roadway conditions, weather conditions, vehicle conditions, and driver conditions are primarily traffic events and/or incidents with some degree of uncertainty. Based on information detected on the state of the traffic environment, drivers select courses of action that may or may not change traffic operation. Driver actions can include slowing down, accelerating, passing a vehicle, turning, etc. Under such circumstance, subsequent studies have also applied the principles behind situation awareness into driving in traffic $^{25-27}$.

Using the principal ideas underlying the models of cognition adopted by Endsley and considering the variations in traffic operations ${ }^{2,8}$, Driving Situation Awareness (DSA) is defined as the perception of the elements in the road environment within a volume of time and space, the comprehension of their meaning, and the projection of their status in the near future ${ }^{25}$ (Fig. 2). It includes level 1 DSA, level 2 DSA and level 3 DSA, which are briefly described and summarized as follow,

Level 1 DSA: Perception of the traffic elements in the driver-vehicle-road system. For example, a driver needs to know where other vehicles and obstacles are, and dynamics of one's own vehicle.

Level 2 DSA: Comprehension of the current driving situation. For example, a driver must comprehend the appearance of neighboring vehicles indicates certain things about their objectives.

Level 3 DSA: Projection of future traffic status. For example, a driver needs to detect future possible collision in order to act effectively. 


\section{3 Driving cognitive behaviour}

According to the degree of novelty from the perspective of human factors in driving, three driving cognitive behaviors can be differentiated for describing the various mechanisms of driver's processing traffic information; they are skill-based driving behaviour, rule-based driving behaviour and knowledge-based driving behavior.

Knowledge-based driving behaviour is concerned with analytical problem solving based on a symbolic representation, whereas skill-based driving behaviour and rule-based driving behaviour are concerned with perception and action. Perceptual processing is fast, effortless, and proceeds in parallel, whereas analytical problem solving is slow, laborious, and proceeds in a serial fashion.
While considering variations of driving situation awareness, Fig. 3 shows the correlation of driving task and driving cognitive behavior, here we could find the relationship among three interfaces such as ecological interface, adaptive interface and traditional interface.

\subsection{Relationship between driving tasks and driving situation awareness}

The driving task is a hierarchical process, including operational, tactical and strategic task, there are dynamic concurrent driving activities at the three driving tasks. The relationship between the three driving tasks and various levels of driving situation awareness could be further described and explained as follows:

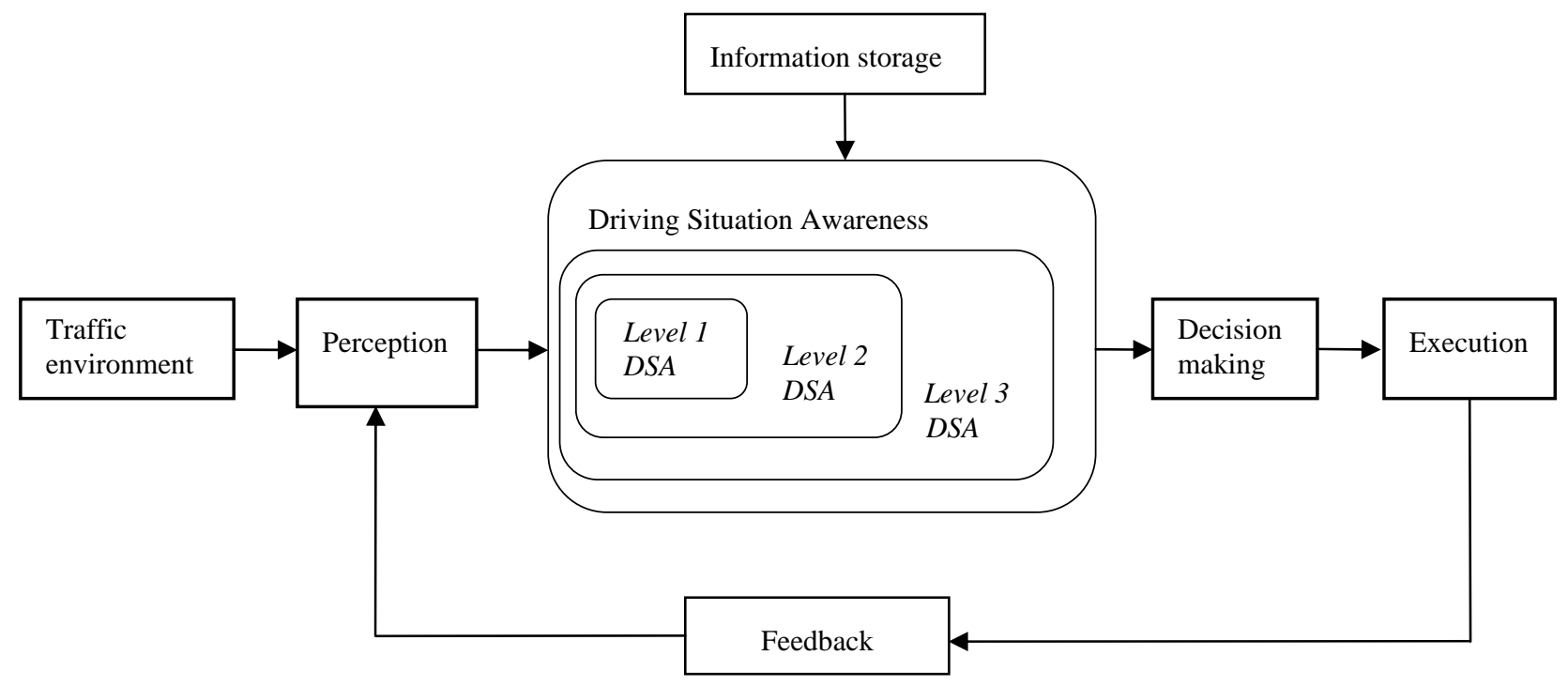

Fig. 2. Driving situation awareness in traffic operation

Operational driving task comprises all those activities that involve second to-second exchange of information and control inputs between the driver and the vehicle (e. g. steering, braking, and shifting). Most driver's control activities are performed automatically with little driver's conscious effort.

Tactical driving task involves the maintenance of common driving situations (e.g., passing in curves and intersections, gap acceptance in overtaking or entering the traffic stream, obstacle avoidance). Information presented to the driver-vehicle system is from traffic control devices, delineation, traffic and other features of the environment, continually changing as the vehicle moves along the road.

Strategic driving task involves route planning and guidance while enroute, for example, correlating directions from in-vehicle map with guide signage in a road network (e. g. Vehicle Information and Communication System, VICS in Japan), setting trip goals (e.g. minimize time, avoid traffic jam). 
At the operational level, drivers are engaged in actions upon vehicle actuators in order to maintain stable vehicle control. This type of task requires Level 1 DSA on semi-automatic processes to ensure that the operations are performed appropriately. Level 2 DSA may be involved if the automatic processes "generate error messages”.

At the tactical level, there is a high requirement for Level 1 and 2 DSA to facilitate local maneuvering of the vehicle in traffic streams, detecting appropriate environmental cues, and comprehending the driving situation. Tactical tasks also require short span projection of the driving environment, probably less than the extensive projection required for strategic driving tasks (Level 3 DSA).

At the strategic level, when navigational plans are formulated, there is a high requirement for Level 3 DSA. At the time of execution, the strategic plan involves elements of Level 2 DSA, in terms of perceptual integration and comprehension. There is also a small contribution from Level 1 DSA, since Level 1 DSA is the basis for the other two levels of DSA ${ }^{24,26}$.

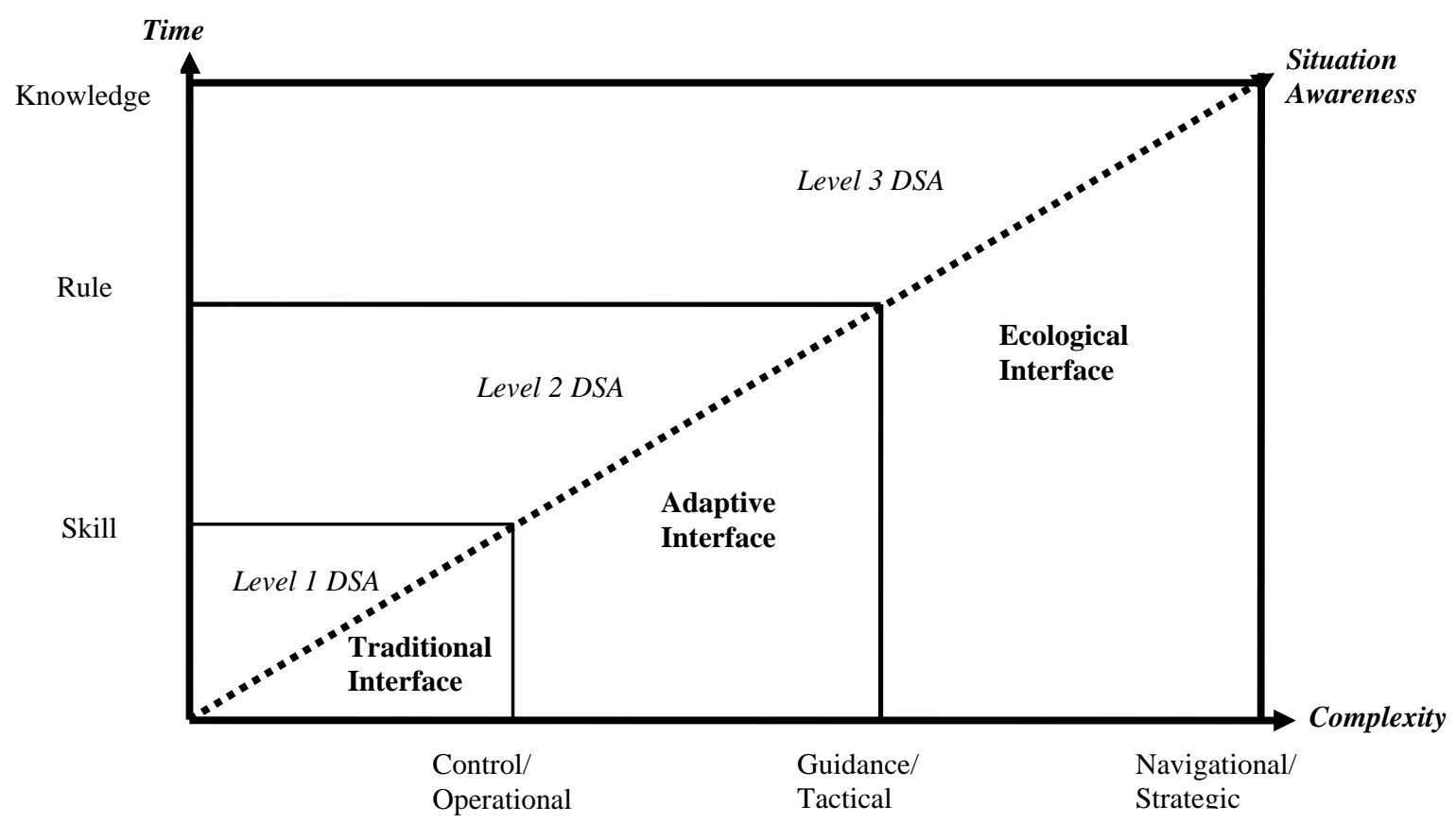

Fig. 3. Correlation of driving task and driving cognitive behaviour

\section{Ecological Function Allocation in Intelligent Driver Interface}

In order to raise driving dependability and enhance intelligent transportation system safety ${ }^{29}$, it is very important to determine what driver to do and what invehicle support systems to execute. While developing the advanced vehicle with driver assistant system, function allocation in driver-vehicle interface will be becoming a vital problem which needs to solve, it concerns with different design decisions that determine reasonably which functions are to be achieved by driver and which are to be performed by in-vehicle support system. In this configuration, optimal function modification and reallocation among driver, in-vehicle support systems and vehicle will depend mainly on the 6 items: (i)real-time traffic situation, (ii)driving cognitive behaviour,(iii)driving tasks, (iv)driving situation awareness, (v)role of in-vehicle support systems , and (vi)different ways of interactive communications.

The main contents of the 6 items could be further explained as follows, 
(i)Real-time traffic situation refers to familiar traffic events, unfamiliar but anticipated traffic events, unfamiliar and unanticipated events,

(ii)Driving cognitive behaviour refers to skill-based driving behaviour, rule-based driving behaviour, knowledge-based driving behaviour,

(iii)Driving tasks involve navigation/strategic level, guidance/tactical level, control/operational level,

(iv)Driving situation awareness refers to perception of the traffic elements, comprehension of the current driving situation, projection of future traffic status,

(v)In-vehicle support systems refer to in-vehicle information acquisition system, in-vehicle information analysis system and in-vehicle information process system,

(vi)Different ways of interactive communications refers to driver, in-vehicle support system and advanced vehicles with intelligent traffic information network environment.

\subsection{Principals of intelligent driver interface design}

While navigation decision making can be largely memory-driven, requiring little if any new information, guidance decision making and control decision making are based on the immediate driving environment and can be considered as mainly data-driven.

Based on the theoretical foundation of ecological interface design that set forth by Vicente and Rasmussen $^{3,6,10-11}$, ecological function allocations in intelligent driver interface consists of four general principles, each corresponding to a specific design requirement.

Principal 1: to support interaction via time-space signals, the driver should be able to act directly on the display, and the structure of the displayed information should be isomorphic to the part-whole structure of movements.

Principal 2: to provide a consistent one-one mapping between the driving domain constraints and the cues or signs provided by the interface.

Principal 3: to represent the driving domain in the form of an abstraction hierarchy to serve as an externalized mental model that will support knowledge-based problem solving.

Principal 4: navigation/strategic decision making can generally be done while driving as time permits from minutes to hours. Guidance/tactical decisions are considered to take place in seconds while control/operational decision require only milliseconds to execute.

Excluding the above four principals, the key driving behaviour shaping factors and disturbances of in-vehicle support systems should considered while conducting function modification and reallocation among driver, invehicle support systems and advanced vehicle ${ }^{25,29}$. In this sense, we might termed such new function allocation with "who does what and when and how" consideration as ecological function allocation, it is third one that is dynamic, interactive and real time, especially it has intelligent characteristics.

In all phases during the period of intelligent driver interface design, the ecological function allocation is largely used to satisfy all requirements of intelligent transportation system safety. Of course, the traditional function allocation and adaptive function allocation are also used in the earlier design phases for summarizing the intra- and inter-relationships of design variables. Fig. 4 shows a systematic structure for 3 types of function allocations for intelligent driver interface design, it is based on the comprehensive considerations of relevant ergonomics knowledge, human factors design principles and manufacturing practices. Especially ecological function allocation is able to provide more flexible design decision for satisfaction all requirements of intelligent driver interface.

\subsection{Process of intelligent driver interface design}

We combine both fundamental ergonomics knowledge with ecological interface design principles, the design and location of the seat, steering wheel, pedal, instrument panel and other new in-vehicle devices are optimized, so we create the intelligent driver interface for the advanced vehicles. The systematic design process in an integrated way consists of five stages:

(i)driving tasks analysis and drivers survey, 
(ii)measurement of driving situation awareness so that reveal that traffic events that are unfamiliar and unanticipated pose the greatest threat to vehicle safety,

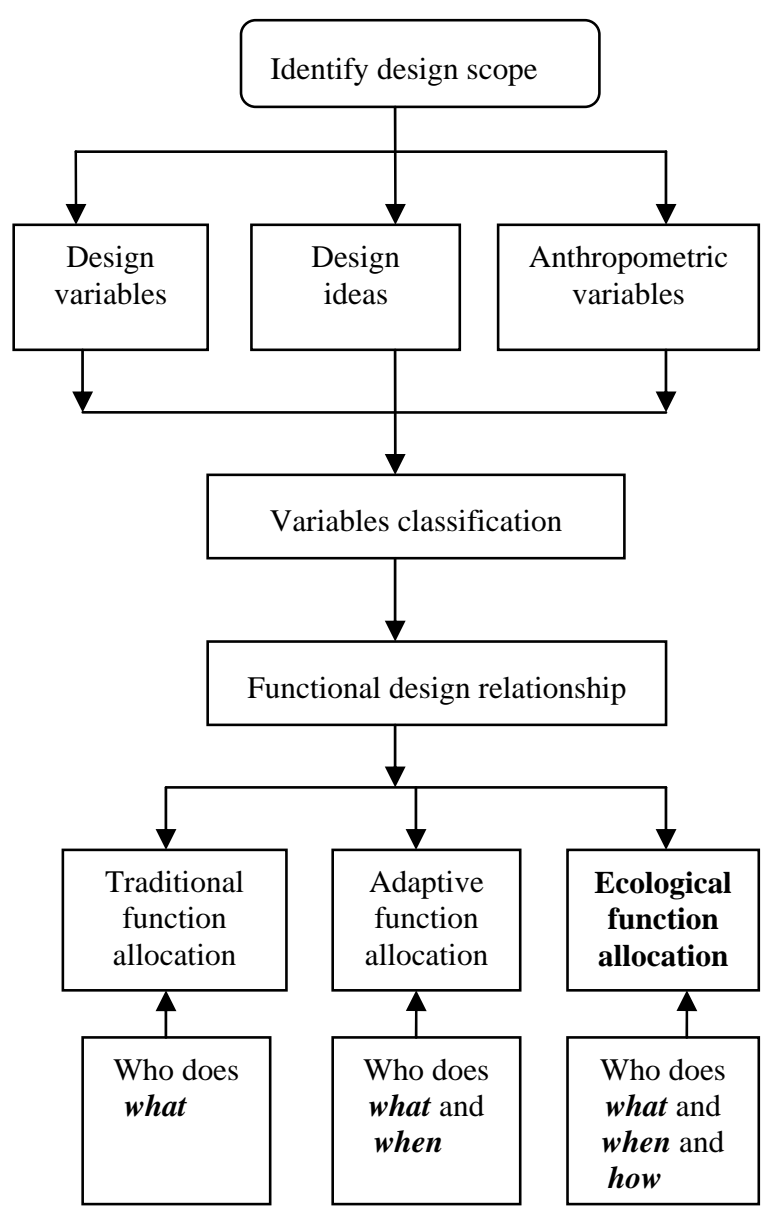

Fig. 4. Systematic design structure for function allocations

(iii)correlation analysis of driving tasks and driving cognitive behaviour,

(iv)development of functional design relationship (intrarelationship analysis of ergonomic, mechanical and aesthetic design variables),

(v)integration and design of human factors in drivervehicle interface.

\section{Conclusions and Future Works}

While the in-vehicle support systems(ISSs) could improve traffic safety, efficiency and comfort, they represent a new frontier in traffic system research and development. Specifically, the relationships between various ISSs design parameters and the driver's ability to effectively and comfortably use ISSs has not been extensively studied. In fact, some past research results are often inappropriate when applied to ISSs design situations because of differences in driver populations, driver expectations, driving tasks, design constraints, design tradeoffs, and driver capabilities and limitations. Our research is intend to provide a new idea about how to address growing information gap between the diverse status of in-vehicle support systems and the availability of human factors design criteria that can be used during the ISSs design process.

The analysis of human factor and safety in drivervehicle interface is expecting to apply ergonomics theory into the design of in-vehicle support systems, a highlighted example is internet-based in-vehicle support systems that have become widespread in recent years. In addition, ergonomics principles are applicable to the broad range of information included routing and guidance information, signing, safety and warning information, as well as motorist services. It will be expecting that much more effective, comfortable and safe ISSs will appear in traffic community if manmachine system characteristics could be considered properly during all phase of ISSs development. In this sense, we propose a conceptual framework for ecological function allocation by "who does what, when and how" consideration during the design process of intelligent driver interface. The primary objective of our research is to explore ecological function allocation and optimization matching solution that will be able to apply into human-machine interface design for intelligent vehicles, it is expecting to address the impact of driver interfaces layouts, traffic information types, and driving behavioral factors on the advanced vehicles design and development.

\section{Acknowledgements}

This research was supported in part by Alexander von Humboldt Foundation (1072467), National Nature Science Foundation of China (50878023), and the Scientific Research Foundation for the Returned Overseas Chinese Scholars of Ministry of Education, P. R. China. 


\section{References}

1 J. Hoc, From human-machine interface to humanmachine cooperation, Ergonomics, 2000 43(7), 833-843.

2 M. R. Endsley and D. B. Kaber, Level of automation effects on performance, situation awareness and workload in a dynamic control task, Ergonomics, 42(1999) ,462-492

3 N. Naito, J. Itoh, K. Monta and M. Makino, An intelligent human-machine system based on an ecological interface design concept,Nuclear Engineering and Design, 154 (1995) 97108

4 J. Rasmussen, Skills, rules, knowledge; signals, signs, and symbols, and other distinctions in human performance models. IEEE Transactions on Systems, Man and Cybernetics, 13(1983), 257-266.

5 T. B. Sheridan, Function allocation: algorithm, alchemy or apostasy? International Journal of Human-Computer Studies 52(2000), 203-216.

$6 \mathrm{~J}$. Rasmussen and K. J. Vicente,Coping with human errors through system design: implication for ecological interface design, Int. J. Man-Machine Studies,31(1989),517-534

7 F. Nachreiner, P. Nickel and I. Meyer, Human factors in process control systems: the design of human-machine interfaces, Safety Science , 44 (2006) 5-26

8 M. R. Endsley, B. Bolte and D. G. Jones, Designing for Situation Awareness: An approach to human-centered design(Taylor \& Francis,London,2003)

9 T. Inagaki,Adaptive automation for comfort and safety,International Journal of ITS Research, 1(2003),3-12 10 K. J. Vicente, Ecological Interface Design: Progress and challenges. Human Factors, 44(2002), 62-78

11 C. M. Burns and J. R. Hajdukiewicz, Ecological Interface Design(CRC Press, Boca Raton,2004)

12 B. Peacock and W. Karwowski, Automotive ergonomics(Taylor \& Francis, London, 1993)

13 C. Little, The Intelligent Vehicle Initiative: Advancing "Human-Centered" Smart Vehicles, Public Roads, 61(2) (1997), 18-25

14 A. R. Hale, J. Stoop and J. Hommels,Human error models as predictors of accident scenarios for designers in road transport systems, Ergonomics,33(1990),1377-1387

15 N. Y. Ian, Ergonomics and safety of intelligent driver interfaces(Lawrence Erlbaum Associates Inc., New Jersey, 1997)

16 M. G. Flyte, The safety design of in-vehicle information and support system: the human factors issues, International journal of vehicle design, 16(1995), 158-169
17 T. A. Ranney, Models of driving behaviour: A review of their evolution, Accident analysis and prevent, 26(1994), 733-750

$18 \mathrm{~W}$. Barfield and T. A. Dingus, Human factors in intelligent transportation systems(Lawrence Erlbaum Associates, Inc. New Jersey, 1997)

19 R. Sukthankar, J. Hancock, C. Thorpe, Tactical-level Simulation for Intelligent Transportation Systems,Mathematical and Computer Modelling, 27(1998), 19-24.

20 W. J. Horrey, C. D. Wickens and K. P. Consalus, Modeling Drivers' Visual Attention Allocation While Interacting With In-Vehicle Technologies, Journal of Experimental Psychology: Applied, 12(2006),67-78

$21 \mathrm{~W}$. H. Wang, A digital driving system for smart vehicle,IEEE Intelligent Systems, 17(5) (2002), 81-83.

22 A. Amditis, K. Pagle, S. Joshi, and E. Bekiaris,DriverVehicle-Environment monitoring for on-board driver support systems: Lessons learned from design and implementation,Applied Ergonomics, 41 (2010) 225-235

23 L. Takayama and C. Nass,Driver safety and information from afar: An experimental driving simulator study of wireless vs. in-car information services, Int. J. HumanComputer Studies 66 (2008) 173-184

24 R. Q. Ma, D. B. Kaber, Situation awareness and workload in driving while using adaptive cruise control and a cell phone, International Journal of Industrial Ergonomics, 35 (2005) 939-953

25 W. H. Wang, Driving Behaviour Theory and Method in Road Transport System (Science Press, Beijing, 2001)

26 M. L. Matthews, D. J. Bryant, R. D. Webb and J. L. Harbluk, Model for situation awareness and driving. Transportation Research Record, 1779 (2001) 26-32.

27 W. H. Wang, Incident Tree Model and Incident Tree Analysis Method for Quantified Risk Assessment: an indepth accident study in traffic operation, Safety Science, $\mathbf{4 8}$ (2010) 1248-1262

28 W. Piechulla, C. Mayser, H. Gehrke and W. Konig,Reducing drivers mental workload by means of an adaptive man-machine interface,Transportation Research Part F 6 (2003) 233-248

29 W. H. Wang,H Bubb,K. Ikeuchi,Q Cao,Measurement of dangerous traffic conditions through driving dependability analysis, Journal of Scientific \& Industrial Research,69(2010) 254-258

30 W. B. Rouse, Design for success: a human centered approach to designing successful production and systems(Wiley,Newyork,1991) 